\title{
Call for Papers for ICGA-91
}

\section{The Fourth International Conference on Genetic Algorithms}

\author{
July 13-16, 1991 \\ University of California at San Diego
}

The Fourth International Conference on Genetic Algorithms (ICGA-91), will be held on July 13-16, 1991 at the University of California - San Diego in La Jolla, CA. This meeting brings together an international community of scientists from academia and industry interested in algorithms suggested by the evolutionary process of natural selection. Topics of particular interest include: Holland's Genetic Algorithm and Classifier Systems, machine learning and optimization using these systems, and their relations to other learning paradigms (e.g., connectionist networks). Papers discussing how genetic algorithms and classifier systems are related to biological modeling issues (e.g., evolution of nervous systems, computational ethology, artificial life) are encouraged. Papers describing significant, unpublished research in these areas are solicited. Authors must submit four (4) complete copies of their paper by February 1, 1991 to the Program Co-Chair:

Dr. Richard K. Belew

Computer Science \& Engr. Dept. (C-014)

Univ. California - San Diego

La Jolla, CA 92093

Electronic submissions (LaTeX source only) can be mailed to rik@cs.ucsd.edu. Papers should be no longer than 10 pages, single spaced, and printed using $12 \mathrm{pt}$. type. All papers will be subject to peer review. Evaluation criteria include the significance of results, originality, and the clarity and quality of the presentation.

\section{Important Dates:}

February 1, 1991:

March 22, 1991:

May 6, 1991:

July 13-16, 1991:
Submissions must be received

Notification mailed to authors

Revised, final camera-ready paper due

Conference dates

\section{ICGA-91 Conference Committee:}

Conference Co-Chairs: Kenneth A. De Jong, George Mason University

J. David Schaffer, Philips Labs

Vice Chair:

David E. Goldberg, Univ. of Illinois at Urbana-Champaign

Program Co-Chairs:

Publicity Chair:

Financial Chair:

Richard K. Belew, Univ. of California at San Diego

Lashon B. Booker, MITRE

Local Arrangements:

David E. Goldberg, Univ. of Illinois at Urbana-Champaign Gil Syswerda, BBN

Richard K. Belew, Univ. of California at San Diego 